\title{
Effect of soil textural characteristics on longitudinal dispersion in saturated porous media
}

\author{
Mojtaba G. Mahmoodlu ${ }^{1,2^{*}}$, Amir Raoof ${ }^{2}$, Martinus Th. van Genuchten ${ }^{2,3}$ \\ ${ }^{1}$ Faculty of Agriculture and Natural Resources, Gonbad Kavous University, Gonbad Kavous, Iran. \\ 2 Department of Earth Sciences, Utrecht University, Utrecht, Netherlands. \\ ${ }^{3}$ Center for Environmental Studies, CEA, São Paulo State University Rio Claro, Brazil. \\ *Corresponding author. E-mails: m.g.mahmoodlu@gmail.com / mmahmoodlu@gonbad.ac.ir
}

\begin{abstract}
This study focuses on the effects of soil textural heterogeneity on longitudinal dispersion under saturation conditions. A series of solute transport experiments were carried out using saturated soil columns packed with two filter sands and two mixtures of these sands, having $d_{50}$ values of $95,324,402$, and $480 \mu \mathrm{m}$, subjected to four different steady flow rates. Values of the dispersion coefficient $(D)$ were estimated from observed in-situ distributions of calcium chloride, injected as a short nonreactive tracer pulse, at four different locations $(11,18,25,36 \mathrm{~cm})$. Analyses of the observed distributions in terms of the standard advection-dispersion equation (ADE) showed that $D$ increased nonlinearly with travel distance and higher Peclet numbers + . The dispersion coefficient of sand sample S1 with its largest average particle size $\left(d_{50}\right)$ was more affected by the average pore-water velocity than sample S4 having the smallest $d_{50}$. Results revealed that for a constant velocity, $D$ values of sample S1 were much higher than those of sample S4, which had the smallest $d_{50}$. A correlation matrix of parameters controlling the dispersion coefficient showed a relatively strong positive relationship between $D$ and the Peclet number. In contrast, almost no correlation was evident between $D$ and porosity as well as grain size. The results obtained with the four sandy matrices were consistent and proved that the dispersion coefficient depends mainly on the particle size.
\end{abstract}

Keywords: Solute dispersion; Soil textural heterogeneity; Saturated columns; Peclet number; Flow velocity; Travel distance.

\section{INTRODUCTION}

Solute dispersion is a key part of solute transport by affecting such processes as subsurface pollutant transport, leaching of pesticides and nutrients from the soil root zone, remediation of contaminated sites, ion exchange chromatography, and many other applications in the soil, agricultural, hydrogeologic, petroleum, and chemical engineering disciplines (e.g., Bear, 1988; Delgado, 2006; Fetter, 2008; Godoy et al., 2019; Lewis and Sjostrom, 2010; Nimbi and Powers, 2003). The problem of solute dispersion has attracted much interest from the early 1900 s onward. However, it gathered much more attention and became a broader topic only after the 1950 s, gathering many studies on with hydrodynamic dispersion and miscible displacement. Many or most previous studies investigated dispersion in fully saturated porous media (e.g., Bear, 1988; Cui et al., 2019; Goldobin and Maryshev, 2017; Saffman, 1959; Scheidegger, 1974). Their findings suggest that the dispersion coefficient $(D)$ increases more or less linearly with the fluid phase velocity and the distance travelled, while also being affected by the size and shape of particles making up the porous medium, media heterogeneity and anisotropy, and pore system tortuosity, as confirmed further by many recent studies (e.g., Bandai et al., 2017; Bromly et al., 2007; Delgado, 2006; Hunt et al., 2011; Poulsen et al., 2008; Pugliese et al., 2013; Sharma and Poulsen, 2010).

Despite the above and many other studies, consistent and comprehensive data sets to establish links between solute dispersion and soil textural characteristics remain limited. The main objective of this study was to investigate the role of soil textural properties on solute dispersion under saturated conditions. For this purpose, we compared tracer breakthrough through four sandy matrices: two sandy matrices (coarse and fine) exhibiting narrow pore size distributions, and two mixtures of these sandy mixtures exhibiting intermediate particle sizes and bimodal distributions. For these matrices, we determined solute breakthrough curves (BTCs) by deriving total resident concentrations from the bulk soil electrical conductivity measured with four-electrode salinity probes at several locations along columns packed submitted to saturated flow. Values of the parameters in the governing advection-dispersion equation (ADE) were estimated by fitting analytical solutions of the transport equation to the observed BTCs. Solute mixing in the saturated sands will be discussed in terms of the value of $D$ as a function of the pore-water velocity, the transport distance, the particle Peclet number, and soil texture (particle size distribution, i.e., grain size mean heterogeneity).

\section{MATERIALS AND METHODS Laboratory experiments Sand properties}

For this study we used two different filter sands (a relatively coarse sand sample S1 and a relatively fine sand sample S4) together with two mixtures (samples S2 and S3) of sands S1 and S4. Sample S2 was a mixture (in weight) of $80 \%$ of S1 and $20 \%$ of S4, and sample S3 was a mixture of $60 \%$ S1 and $40 \%$ S4. X-ray micro-computed tomography images of the four samples are shown in Fig.1a. The images of sand mixtures S2 and $\mathrm{S} 3$ exhibited a relatively significant degree of heterogeneity in terms of particle.

The sands originated from a river bed in Papendrecht, Netherlands (Filtersand, Filcom, Netherlands) and were sieved to different retained sizes as given in Table 1. The average particle size $\left(d_{50}\right)$ varied from $90 \mu \mathrm{m}$ to $480 \mu \mathrm{m}$ (from sands $\mathrm{S} 4$ to $\mathrm{S} 1$ ). 
a)

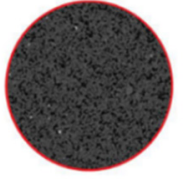

S4

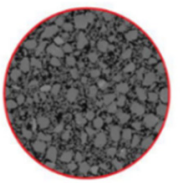

S3

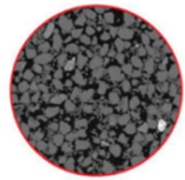

S2

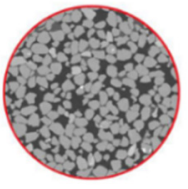

S1 b)

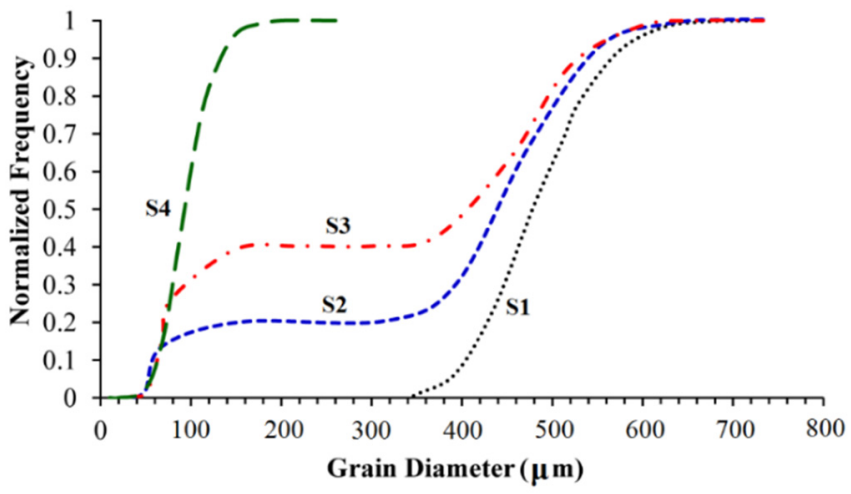

Fig. 1. MicroCT scans of the sand samples (a), and their particle size distributions (b). Samples S1 (black dotted line) and S4 (green dashed line) represented relatively coarse and fine sands, respectively. Sample S2 (blue dashed line) is a mixture of $80 \%$ of S1 and $20 \%$ of S4, and sand sample S4 (red dash-dotted line) is a mixture of $60 \% \mathrm{~S} 1$ and $40 \% \mathrm{~S} 4$.

X-ray fluorescence analyses showed that more than $96 \%$ of the sand grains consisted of silica $\left(\mathrm{SiO}_{2}\right)$.

Coefficients of uniformity $\left(\mathrm{Cu}=d_{60} / d_{10}\right)$, curvature $(C c=$ $\left.d_{30}{ }^{2} / d_{10} \times d_{60}\right)$, and sorting $\left(S o=\left(d_{75} / d_{25}\right)^{1 / 2}\right)$ were estimated for all sands (Table 1). Since $C u$ was less than 4.0 for sands S1 and $\mathrm{S} 4$, these two samples can be considered to be uniformly graded by containing essentially identical particle sizes. Sand S1 was the most uniform. We further included in Table 1 the coefficient of curvature $(C c)$ as another useful measure of the shape of the particle size distribution curve. This coefficient is sometimes used to differentiate the sorting degree of a soil. Sands S2 and S3 clearly showed lesser degrees of sorting as reflected by their bimodal grain size distributions (Fig. 1). In our study, we used sand $\mathrm{S} 1$ as the main sand in view of its low $\mathrm{Cu}, \mathrm{So}$, and $d_{50}$ values. Mixing different fractions of main sand S4 with sand S1 created far more heterogeneous media (Fig. 1). We expect two mixtures with intermediate particle size and bimodal pore size distributions to exhibit different transfer properties.

\section{Experimental columns}

Fig. 2 shows a schematic of the experimental setup used in our study. Plexiglass cylinders of $36 \mathrm{~cm}$ length and $9.0 \mathrm{~cm}$ internal diameter were used to construct the columns, which contained stainless steel lids with a port at its inlet and outlet faces. Three measuring probes at depths of 11,18 and $25 \mathrm{~cm}$ and at the outlet, at $36 \mathrm{~cm}$, were used to obtain solute concentrations utilizing the measured electrical conductivity (EC). A picture of the experimental laboratory setup is shown in Fig. 3.

Sand columns S1, S2, S3, and S4 were packed to porosity values of approximately $0.38,0.34,0.32$, and 0.40 , respectively. After packing, the sand columns were flushed with $\mathrm{CO}_{2}$ for a few hours to replace air from the soil pore spaces, thus ensuring as saturated as possible conditions once filled with water. Next, the columns were gradually saturated using deionized water from the bottom using a peristaltic pump (Ismatec, Switzerland). Although peristaltic pumps deliver pulsated flow, the relatively large diameter of the columns together with their

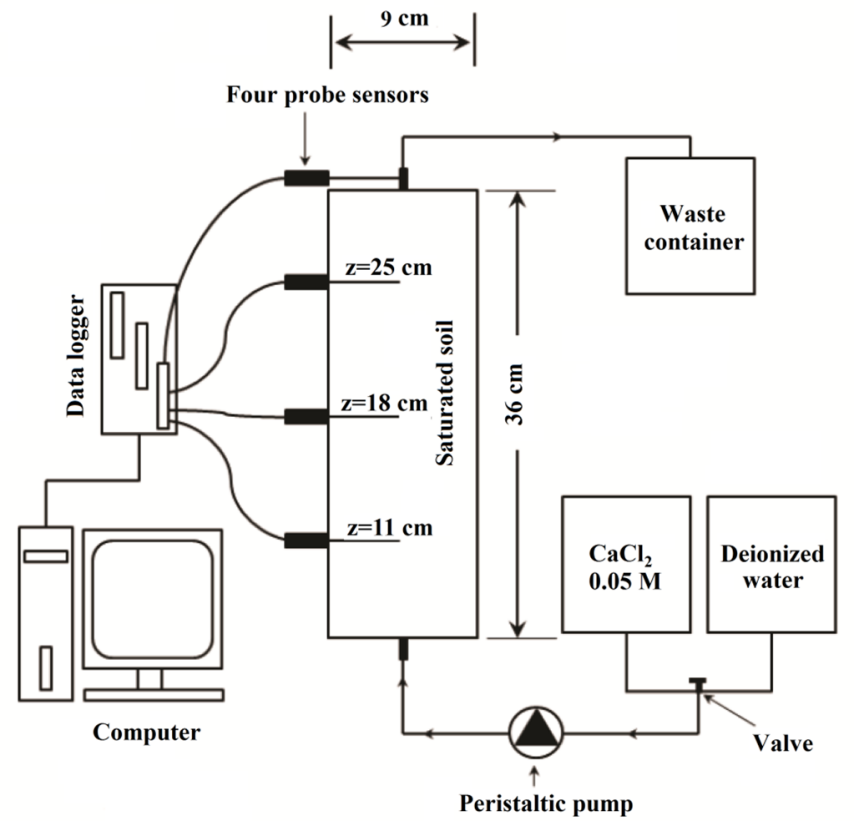

Fig. 2. Schematic of the experimental setup and sand columns used in this study.

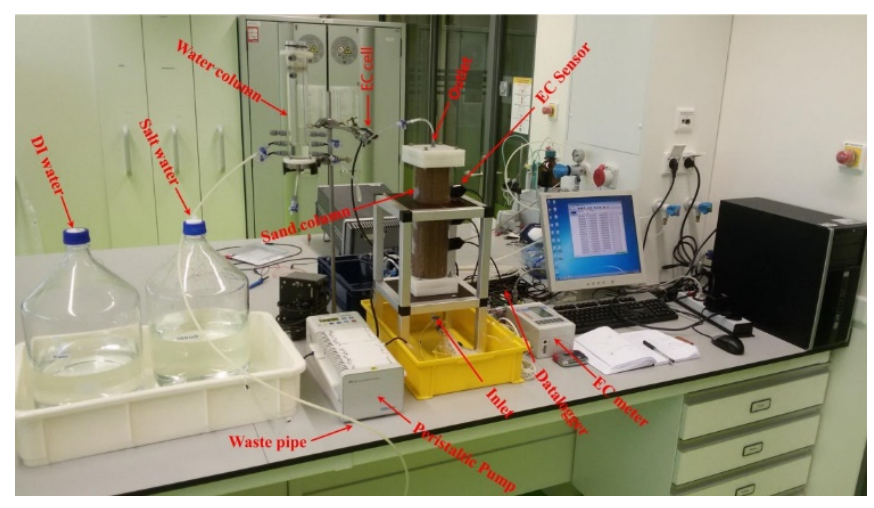

Fig. 3. Picture of the experimental laboratory setup.

Table 1. Selected properties of the natural sands used in this study ${ }^{1}$.

\begin{tabular}{|c|c|c|c|c|c|c|c|c|c|c|c|c|}
\hline Sample & $\begin{array}{l}d_{10} \\
(\mu \mathrm{m})\end{array}$ & $\begin{array}{l}d_{25} \\
(\mu \mathrm{m})\end{array}$ & $\begin{array}{l}d_{30} \\
(\mu \mathrm{m})\end{array}$ & $\begin{array}{l}d_{50} \\
(\mu \mathrm{m})\end{array}$ & $\begin{array}{l}d_{60} \\
(\mu \mathrm{m})\end{array}$ & $\begin{array}{l}d_{75} \\
(\mu \mathrm{m})\end{array}$ & ${ }^{2} \mathrm{Cu}$ & ${ }^{3} \mathrm{Cc}$ & ${ }^{4}$ So & ${ }^{5} \phi$ & $\mathrm{pH}$ & $\begin{array}{l}{ }^{6} \mathrm{TOC} \\
\left(\mathrm{g} \mathrm{m}^{-3}\right)\end{array}$ \\
\hline $\mathrm{S} 1$ & 400 & 440 & 445 & 480 & 500 & 525 & 1.25 & 0.99 & 1.09 & 0.38 & 7 & $<5.0 \times 10^{-20}$ \\
\hline${ }^{7} \mathrm{~S} 2$ & 60 & 380 & 390 & 402 & 460 & 480 & 7.67 & 5.51 & 1.12 & 0.34 & 7 & $<5.0 \times 10^{-20}$ \\
\hline${ }^{8} \mathrm{~S} 3$ & 60 & 70 & 90 & 324 & 440 & 500 & 7.33 & 0.31 & 2.67 & 0.32 & 7 & $<5.0 \times 10^{-20}$ \\
\hline $\mathrm{S} 4$ & 60 & 70 & 80 & 95 & 100 & 115 & 1.67 & 1.07 & 1.28 & 0.40 & 7 & $<5.0 \times 10^{-20}$ \\
\hline
\end{tabular}

${ }^{1}$ Technical data sheet of filter sands (Filcom, Papendrecht, Netherlands): ${ }^{2} \mathrm{Cu}$ : Coefficient of uniformity $\left(C u=d 60 / d_{10}\right) ;{ }^{3} C c$ : Coefficient of curvature $\left(C c=d_{30}{ }^{2} / d_{10} \times d_{60}\right) ;{ }^{4} S_{o}$ : Sorting coefficient $\left(S o=\left(d_{75} / d_{25}\right){ }^{1 / 2}\right) ;{ }^{5} \phi$ : Porosity; ${ }^{6}$ TOC: Total organic carbon of dry sample; ${ }^{7} \mathrm{~S} 2=\mathrm{S} 1(0.8)$ and $\mathrm{S} 4(0.2)$; ${ }^{8} \mathrm{~S} 3=\mathrm{S} 1(0.6)$ and $\mathrm{S} 4(0.4)$. 
relatively long lengths $(36 \mathrm{~cm})$ attenuated the pulsed input and provided a relative continuous form of flow. We subsequently established constant flow rates, ranging from 0.9 to $5.3 \mathrm{~cm}^{3}$ $\mathrm{min}^{-1}$. For the outlet, we implemented a fixed hydraulic head at the top of the sand columns using a small water container. After reaching fully saturated steady-state flow conditions, we applied a short $\mathrm{CaCl}_{2}$ pulse input having a concentration of 0.05 M (Sigma-Aldrich).

Dynamic measurements of soil moisture, temperature, and EC were carried out over time using several 5TE sensors (Decagon). Three 5TE sensors were inserted horizontally in the columns (Bear, 1988; Bunsri et al., 2008a; Dong et al., 2014; Inoue et al., 2000) via measuring holes. Each probe consisted of three 5.2-cm long, $0.5-\mathrm{cm}$ wide, and $0.17-\mathrm{cm}$ thick prongs. The 5TE sensors provided simultaneous measurements of the electrical conductivity of the bulk medium $\left(E C_{b}\right)$ surrounding the sensors, as well as of the bulk medium dielectric permittivity $\left(\varepsilon_{b}\right)$ for the same volume. Hilhorst (2000) and Scudiero et al., (2012), among others, showed that a linear relationship exists between $E C_{b}$ and the electrical conductivity of pore water $\left(E C_{p}\right)$ :

$$
E C_{p}=\frac{\varepsilon_{p}}{\varepsilon_{b}-\varepsilon_{\sigma b=0}} E C_{b}
$$

where $\varepsilon_{p}$ denotes the real portion of the dielectric permittivity of soil pore water, which is a function of soil temperature, defined as $\varepsilon_{p}=80.3-0.37\left(T_{\text {soil }}-20\right)$, and where $\varepsilon_{\sigma b=0}$ is the real portion of the dielectric permittivity of the soil when the bulk electrical conductivity is zero.

\section{BTC data analysis}

As noted earlier, the hydrodynamic dispersion coefficient $(D)$ depends on a range of microscopic and macroscopic factors such as the fluid flow velocity, the tortuosity of the solute travel path in the medium, soil texture, possible anisotropic conditions, and ionic or molecular diffusion processes. At the macroscopic scale, the classical model for describing non-adsorbing solute transport is the advection-dispersion equation (ADE), considering no water fractionation in the columns (van Genuchten and Wierenga, 1976). The ADE equation reads as follows for a uniformly packed one-dimensional column subjected to constant flow (Bear, 1988):

$$
\frac{\partial C}{\partial t}=D \frac{\partial^{2} C}{\partial x^{2}}-v \frac{\partial C}{\partial x}
$$

where $C$ is the average solute concentration, $x$ is the longitudinal distance, $t$ is time, $D$ is the hydrodynamic dispersion coefficient, and $v$ the average pore-water velocity (the Darcian flow velocity divided by porosity).

Assuming the application of a short solute pulse of duration $t_{o}$ at the inlet (at $x=0$ ) to an initially solute-free semi-infinite profile, the analytical solution for the tracer distribution within the column is given by (Lindstrom et al., 1967):

$C(x, t)=\left\{\begin{array}{lr}C_{o} A(x, t) & 0<t \leq t_{o} \\ C_{o}\left[A(x, t)-A\left(x, t-t_{o}\right)\right] & t>t_{o}\end{array}\right.$

where $C_{0}$ is the input concentration and $A(x, t)$ is a term defined as follow:

$$
\begin{aligned}
& A(x, t)=\frac{1}{2} \operatorname{erfc}\left[\frac{x-v t}{\sqrt{4 D t}}\right]+\sqrt{\frac{v^{2} t}{\pi D}} \exp \left[-\frac{(x-v t)^{2}}{4 D t}\right] \\
& -\frac{1}{2}\left(1+\frac{v x}{D}+\frac{v^{2} t}{D}\right) \exp \left(\frac{v x}{D}\right) \operatorname{erfc}\left[\frac{x+v t}{\sqrt{4 D t}}\right]
\end{aligned}
$$

As shown by several authors (e.g., van Genuchten and Parker, 1984), Eq. (4) is an appropriate solution for the volumeaveraged (resident) concentration within semi-finite systems.

The CXTFIT computer program (Toride et al., 1999) within the STANMOD software (Šimunek et al., 2000; van Genuchten et al., 2012) was used to estimate the parameters $v$ and $D$ in Eq. (4) from the observed concentration BTCs obtained for each transport experiment at each location in the columns. CXTFIT uses a Marquardt-Levenberg type nonlinear least squares fitting procedure to optimize the fit between the observed data and the advection-diffusion equation.

For saturated porous media, hydrodynamic dispersion is known to be a function of diffusion and mechanical dispersion, the latter resulting from local velocity variations, leading to the following general relationship for one-dimensional transport (Bear, 1988; Fetter, 2008; Freeze and Cherry, 1979):

$D=D_{e}+\lambda v^{n}$

where $D_{e}$ is the effective diffusion coefficient, $\lambda$ is the (longitudinal) dispersivity, and $n$ is a mostly empirical parameter. As shown by Eq. (5), the hydrodynamic dispersion includes both ionic or molecular diffusion $\left(D_{e}\right)$ and mechanical dispersion due to solute advection processes $\left(\lambda v^{n}\right)$. The effects of diffusion on hydrodynamic dispersion can be evaluated by using a particle Peclet number $\left(P_{e}\right)$ given by:

$P_{e}=\frac{v d}{D_{e}}$

where $d$ is the mean size of soil particles (Kutílek and Nielsen, 1994; Toride et al., 2003). At relatively low values of the Peclet number $\left(P_{e}<3\right)$, solute dispersion is dominated mostly by molecular diffusion processes, while at high values (e.g., $\left.P_{e}>10\right)$, the contribution of molecular diffusion becomes negligible (Delgado, 2007; Toride et al., 2003). At high Peclet numbers, the exponent $n$ of the mechanical dispersion component is generally very close to unity (Bear, 1988; Bolt, 1979; Scheidegger, 1974; among others). This then reflects a linear increase in hydrodynamic dispersion with the pore-water velocity, at least for ideal media such as glass beads, packed beds, and non-aggregated soils.

\section{RESULTS AND DISCUSSION Breakthrough curves (BTCs)}

The measured BTCs at three different depths (i.e., at 11, 18, $25 \mathrm{~cm}$ ) and of the effluent at $36 \mathrm{~cm}$ are shown in Fig. 4 in terms of normalized concentrations $\left(C / C_{o}\right)$ of $\mathrm{CaCl}_{2}$. The BTCs show a clear dominant Gaussian shape at all depths, characteristic of equilibrium transport with Peclet numbers larger than 1.0. The symmetric shape of the BTCs agrees with many studies using tracer transport in homogenously packed soil columns under saturated conditions (Lee et al., 2014; Toride et al., 2003). Using CXTFIT, we fitted the ADE analytical solution to the observed BTCs to determine values of the pore-water velocity, $v$, and the solute dispersion coefficient, $D$, from which the solute dispersivity, $\lambda$, was calculated. Here we assume that the contribution of molecular diffusion is negligible and that the exponent $n$ in Eq. (5) is equal to one. 
Table 2. Measured and calculated values of the pore-water velocity $(v)$ together with their differences $(\Delta v)$, the dispersion coefficient $(D)$ fitted to the observed BTCs, and dispersivity values $(\lambda)$ for various imposed flow rates $(q)$ and travel distances $(L)$.

\begin{tabular}{|c|c|c|c|c|c|c|c|}
\hline Sample & $\begin{array}{c}q \\
\left(\mathrm{~cm}^{3} \mathrm{~min}^{-1}\right)\end{array}$ & $\begin{array}{c}L \\
(\mathrm{~cm})\end{array}$ & $\begin{array}{l}\text { Measured } v \\
\left.(\mathrm{~cm} \mathrm{~min})^{-1}\right)\end{array}$ & $\begin{array}{c}\text { Fitted } v \\
\left.(\mathrm{~cm} \mathrm{~min})^{-1}\right)\end{array}$ & $\Delta \mathrm{v}$ & $\begin{array}{c}\text { Fitted } D \\
\left(\mathrm{~cm} \mathrm{~min}^{-1}\right)\end{array}$ & $\begin{array}{c}\lambda \\
(\mathrm{cm}) \\
\end{array}$ \\
\hline \multirow{12}{*}{ S1 } & \multirow{4}{*}{2.43} & 11 & 0.091 & 0.079 & 0.012 & 0.003 & 0.034 \\
\hline & & 18 & 0.091 & 0.074 & 0.017 & 0.007 & 0.092 \\
\hline & & 26 & 0.091 & 0.079 & 0.012 & 0.009 & 0.121 \\
\hline & & 36.5 & 0.091 & 0.076 & 0.015 & 0.010 & 0.131 \\
\hline & \multirow{4}{*}{3.90} & 11 & 0.145 & 0.147 & -0.002 & 0.005 & 0.034 \\
\hline & & 18 & 0.145 & 0.143 & 0.002 & 0.012 & 0.083 \\
\hline & & 26 & 0.145 & 0.145 & 0.00 & 0.015 & 0.103 \\
\hline & & 36.5 & 0.145 & 0.150 & -0.005 & 0.018 & 0.119 \\
\hline & \multirow{4}{*}{5.30} & 11 & 0.197 & 0.210 & -0.013 & 0.008 & 0.039 \\
\hline & & 18 & 0.197 & 0.200 & -0.003 & 0.014 & 0.071 \\
\hline & & 26 & 0.197 & 0.198 & -0.001 & 0.018 & 0.091 \\
\hline & & 36.5 & 0.197 & 0.198 & -0.001 & 0.020 & 0.101 \\
\hline \multirow{12}{*}{ S2 } & \multirow{4}{*}{1.55} & 11 & 0.064 & 0.071 & -0.007 & 0.001 & 0.021 \\
\hline & & 18 & 0.064 & 0.068 & -0.004 & 0.004 & 0.056 \\
\hline & & 26 & 0.064 & 0.064 & 0.00 & 0.004 & 0.067 \\
\hline & & 36.5 & 0.064 & 0.061 & 0.003 & 0.005 & 0.079 \\
\hline & \multirow{4}{*}{3.07} & 11 & 0.129 & 0.123 & 0.006 & 0.003 & 0.022 \\
\hline & & 18 & 0.129 & 0.123 & 0.006 & 0.006 & 0.053 \\
\hline & & 26 & 0.129 & 0.124 & 0.005 & 0.008 & 0.066 \\
\hline & & 36.5 & 0.129 & 0.124 & 0.005 & 0.009 & 0.075 \\
\hline & \multirow{4}{*}{4.87} & 11 & 0.204 & 0.206 & -0.002 & 0.005 & 0.023 \\
\hline & & 18 & 0.204 & 0.203 & 0.001 & 0.009 & 0.043 \\
\hline & & 26 & 0.204 & 0.204 & 0.00 & 0.011 & 0.052 \\
\hline & & 36.5 & 0.204 & 0.201 & 0.003 & 0.012 & 0.061 \\
\hline \multirow{12}{*}{ S3 } & \multirow{4}{*}{0.90} & 11 & 0.040 & 0.038 & 0.002 & 0.001 & 0.037 \\
\hline & & 18 & 0.040 & 0.039 & 0.001 & 0.002 & 0.062 \\
\hline & & 26 & 0.040 & 0.039 & 0.001 & 0.003 & 0.079 \\
\hline & & 36.5 & 0.040 & 0.040 & 0.00 & 0.004 & 0.091 \\
\hline & \multirow{4}{*}{2.80} & 11 & 0.125 & 0.115 & 0.010 & 0.003 & 0.026 \\
\hline & & 18 & 0.125 & 0.112 & 0.013 & 0.006 & 0.058 \\
\hline & & 26 & 0.125 & 0.113 & 0.012 & 0.008 & 0.073 \\
\hline & & 36.5 & 0.125 & 0.12 & 0.005 & 0.010 & 0.087 \\
\hline & \multirow{4}{*}{5.00} & 11 & 0.223 & 0.218 & 0.005 & 0.005 & 0.025 \\
\hline & & 18 & 0.223 & 0.220 & 0.003 & 0.012 & 0.053 \\
\hline & & 26 & 0.223 & 0.221 & 0.002 & 0.015 & 0.067 \\
\hline & & 36.5 & 0.223 & 0.220 & 0.003 & 0.018 & 0.081 \\
\hline \multirow{12}{*}{ S4 } & \multirow{4}{*}{2.43} & 11 & 0.091 & 0.067 & 0.024 & 0.002 & 0.014 \\
\hline & & 18 & 0.091 & 0.078 & 0.013 & 0.004 & 0.033 \\
\hline & & 26 & 0.091 & 0.082 & 0.009 & 0.006 & 0.047 \\
\hline & & 36.5 & 0.091 & 0.090 & 0.001 & 0.008 & 0.063 \\
\hline & \multirow{4}{*}{3.90} & 11 & 0.123 & 0.120 & 0.003 & 0.0005 & 0.004 \\
\hline & & 18 & 0.123 & 0.121 & 0.002 & 0.002 & 0.014 \\
\hline & & 26 & 0.123 & 0.118 & 0.005 & 0.003 & 0.027 \\
\hline & & 36.5 & 0.123 & 0.122 & 0.001 & 0.004 & 0.036 \\
\hline & \multirow{4}{*}{5.20} & 11 & 0.129 & 0.125 & 0.004 & 0.0005 & 0.007 \\
\hline & & 18 & 0.129 & 0.123 & 0.006 & 0.002 & 0.022 \\
\hline & & 26 & 0.129 & 0.129 & 0.00 & 0.003 & 0.038 \\
\hline & & 36.5 & 0.129 & 0.128 & 0.001 & 0.004 & 0.049 \\
\hline
\end{tabular}

Fig. 4 shows excellent agreement between the simulated macroscale (continuum scale) BTC's using Eq. (4), and the experimental data. Results of the optimized parameters are provided in Table 2. $R^{2}$ values for regression of the observed versus predicted data for all experiments were very high, approximately 0.99 or closer to unity.
Fig. 5 further shows satisfactory agreement between the estimated pore-water velocities $(v)$ at the column scale, calculated from the measured Darcian flux $(q)$ and porosity $(\phi)$ of the columns (i.e., using $v=q / \phi$ ), versus velocities estimated by fitting the measured BTC's using Eq. (3). 

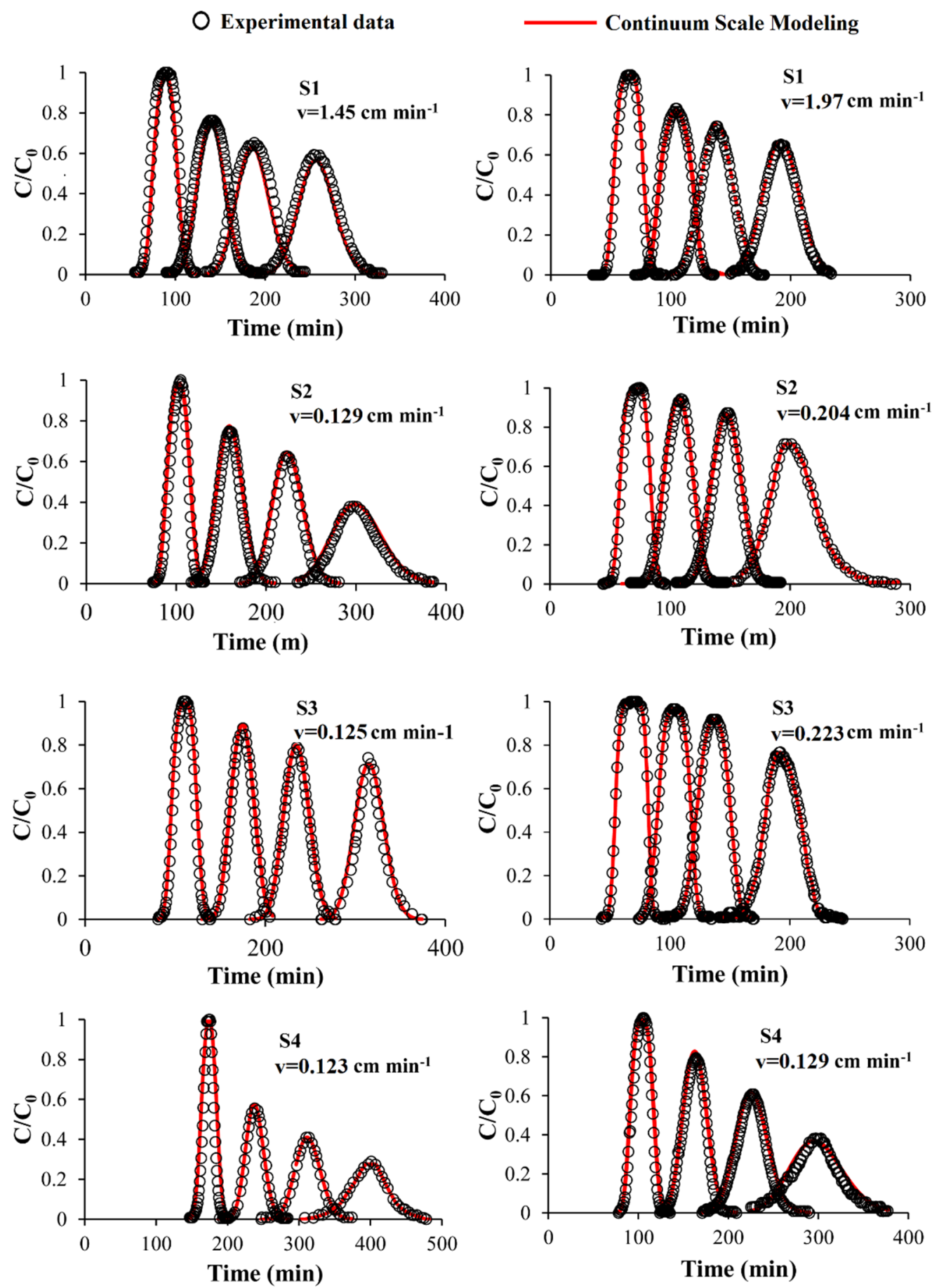

Fig. 4. Measured (open circles) and calculated (lines) breakthrough curves at three different depths $(11,18$, and $25 \mathrm{~cm})$ and the effluent at $36 \mathrm{~cm}$ of the saturated columns. Assuming one dimensional macroscopic flow, Eq. (4) was used for the continuum scale simulations of the BTCs. The curves are for two experiments with each soil sample involving two different flow rates as listed in Table.

\section{Velocity effects}

Fig. 6 shows that the longitudinal dispersion coefficient $(D)$ increases with the pore-water velocity $(v)$ and that for a given velocity, the dispersion coefficient increases with travel distance. Furthermore, results revealed that the dispersion coefficient of sand sample S1, which had the larger mean particle size $\left(d_{50}=480 \mu \mathrm{m}\right)$, was affected considerably by the porewater velocity. In contrast, the dispersion coefficient of sample $\mathrm{S} 4$, having a much smaller mean particle size $\left(d_{50}=95 \mu \mathrm{m}\right)$, was far less affected by the pore-water velocity.
Another way of visualizing the data in Fig. 6 is to plot for each given value of the pore-water velocity the dispersion coefficient as a function of the dispersivity $(\lambda)$. This is done in Fig. 7 , which shows linear relationships between $D$ and $\lambda$, with the slope in all cases being very close to the measured pore-water velocity, as expected. The linear plots in Fig. 7 confirm our assumptions that the effective diffusion coefficient $\left(D_{e}\right)$ in Eq. (5) is very small and can be neglected for all practical purposes $\left(P_{e}>3\right.$ for all sands except S4), and that the exponent $n$ is essentially equal to 1.0. The plots in Fig. 7 further indicate that the dispersivity increases with travel distance. 


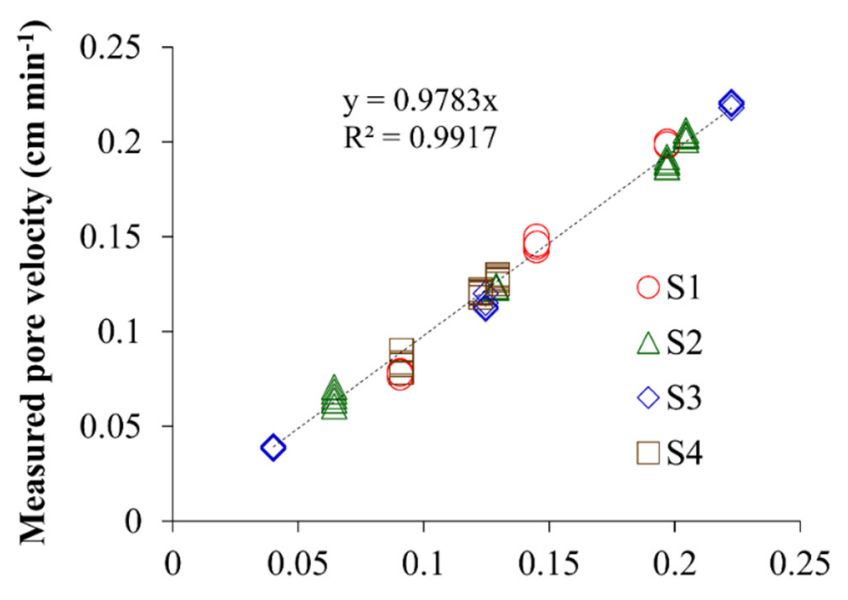

Calculated pore velocity $\left(\mathrm{cm} \mathrm{min}^{-1}\right)$

Fig. 5. Pore-water velocities estimated from the measured BTCs using CXTFIT, versus measured velocities (using a total of 52 data) calculated from the Darcian flux and the porosity.

\section{Effects of travel distance}

Consistent with the results in Figs. 6 and 7, Figs. 8 and 9 show that the macroscale ADE model at different locations provided values for dispersion coefficients and solute dispersivity that increase with travel distance, respectively. The values slowly approach asymptotic values after a travel distance of around 25 or $30 \mathrm{~cm}$. Our results reveal that for a constant velocity, the dispersion coefficient of sand sample S1, having a $d_{50}$ value of $480 \mu \mathrm{m}$, was much higher than the dispersion coefficient of sample S4 having a $d_{50}$ of $95 \mu \mathrm{m}$. These results are consistent with many earlier experimental studies using repacked soils showing a dependency of the longitudinal dispersion coefficient on travel distance and the pore-water velocity (Bromly et al., 2007; Kanzari et al., 2015; Nützmann et al., 2002; Toride et al., 2003; Vanderborght and Vereecken, 2007). Remarkably, Fig. 9 shows that the dispersivity values for the lower travel distances are close to the average grain size for each sample. As the travel distance increased, dispersivity values also increased and became larger than the average grain size for each sand sample.

Despite the dispersion coefficient increasing with the porewater velocity and travel distance, Fig. 8 suggests that $D$ is further affected by grain size and consequently the pore size and its distribution in a porous media. Results show that at a velocity of $0.129 \mathrm{~cm} \mathrm{~min}^{-1}$, the dispersion coefficient of sand sample S4 with a $d_{50}$ of $95 \mu \mathrm{m}$ is much smaller than the $D$ of sand sample S1 having a $d_{50}$ of $480 \mu \mathrm{m}$ at a velocity of 0.197 $\mathrm{cm} \min ^{-1}$.

\section{Effect of Peclet number}

We next calculated the dimensionless Peclet number (Eq. 6) to evaluate the effect of molecular diffusion on the hydrodynamic dispersion. For samples S1 and S2, very high Peclet numbers $\left(P_{e}>10\right)$ were obtained (Fig. 9). This confirms the validity of our assumption that the contribution of molecular diffusion is negligible, and that the empirical constant $n$ is equal to one (Delgado, 2007; Toride et al., 2003).
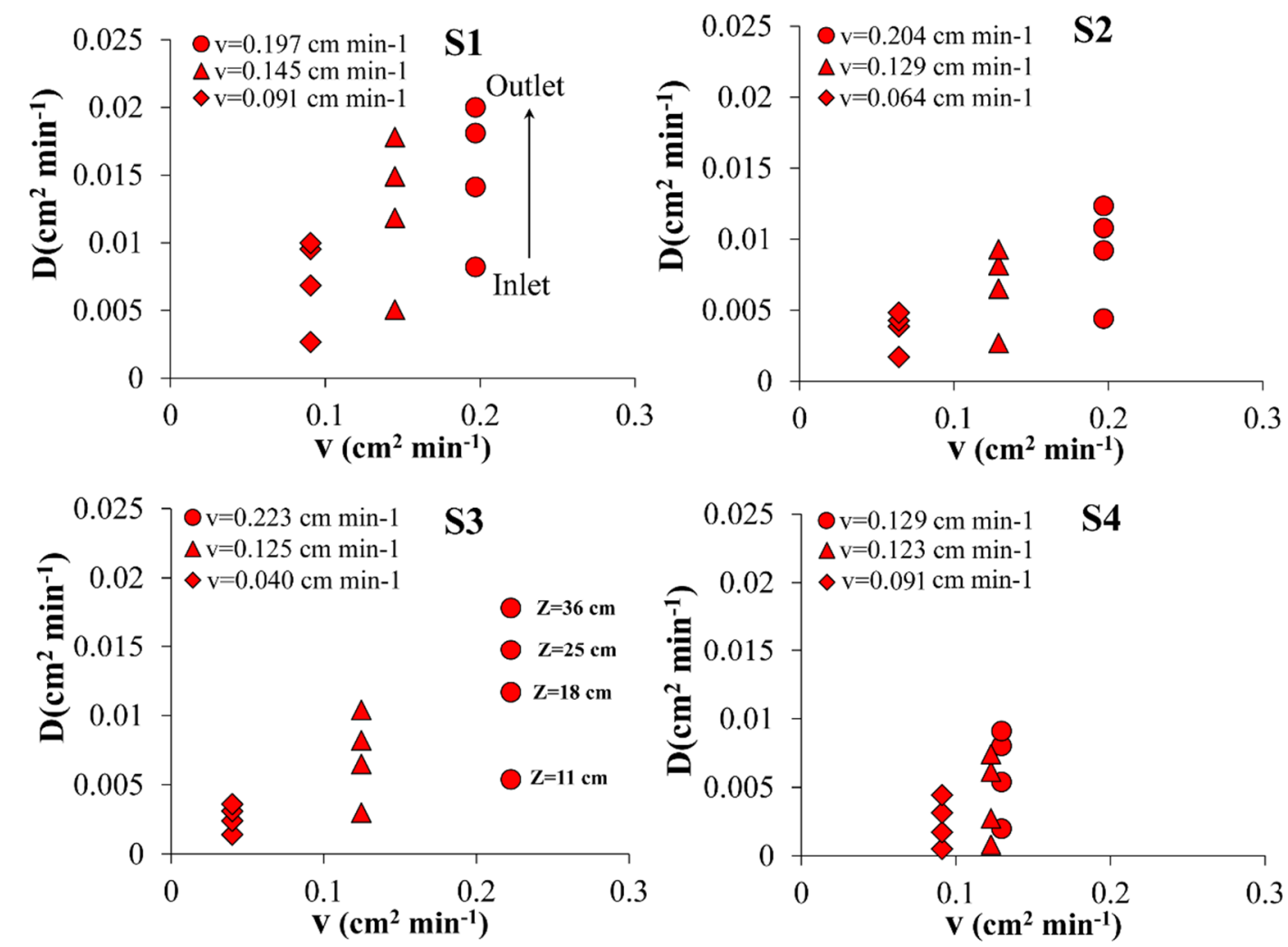

Fig. 6. Plots of the estimated longitudinal dispersion coefficient $(D)$ as a function of the pore-water velocity $(v)$ for the different types of porous media. S1, S2, S3 and S4 have average mean particle sizes of 480, 402, 324, and $95 \mu \mathrm{m}$, respectively. 

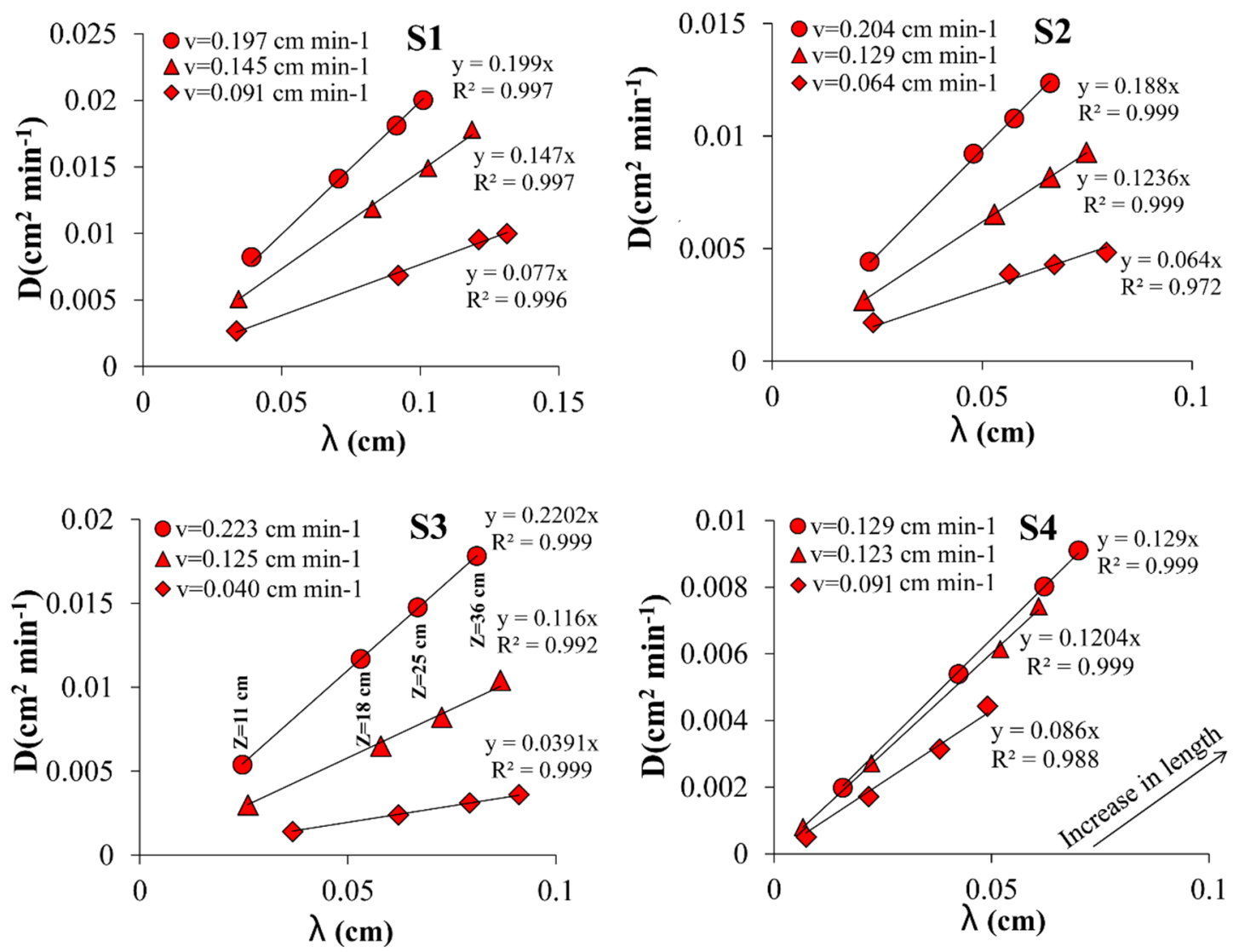

Fig. 7. Estimated longitudinal dispersion coefficient $(D)$ as a function of the dispersivity $(\lambda)$ at given pore velocities $(v)$.
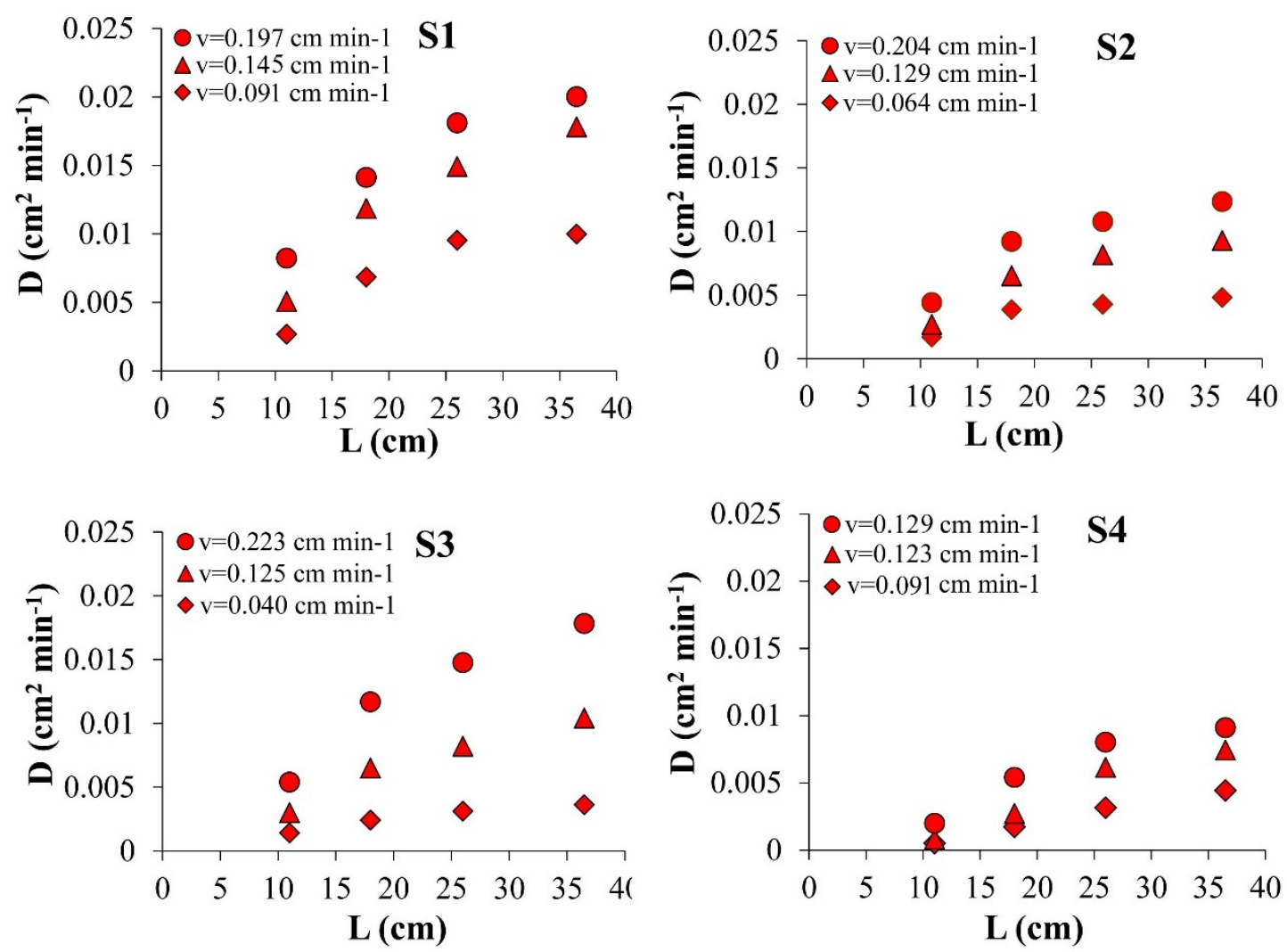

Fig. 8. Effect of travel distance $(L)$ on the longitudinal solute dispersion coefficient $(D)$ for different pore-water velocity $(v)$ values. 


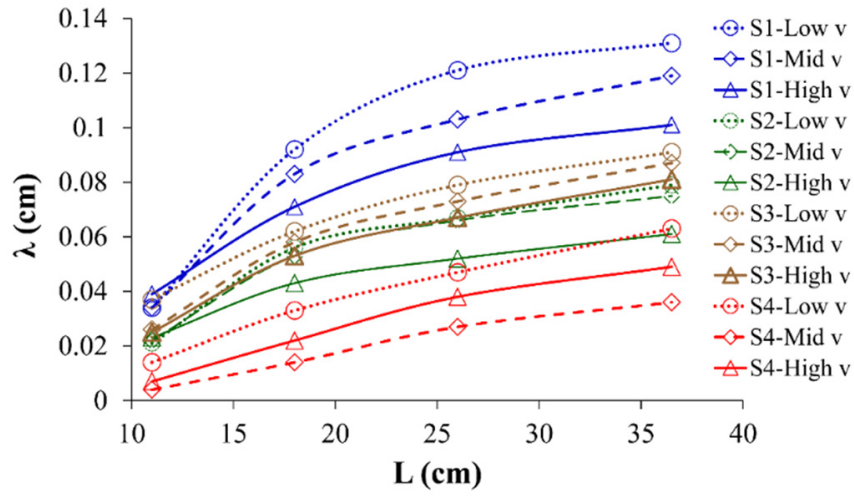

Fig. 9. Effect of travel distance $(L)$ on the solute dispersivity $(\lambda)$ for different pore-water velocity $(v)$ values, "Low v", "Mid v" and "High v" refer to low, medium and high flow rates, respectively.

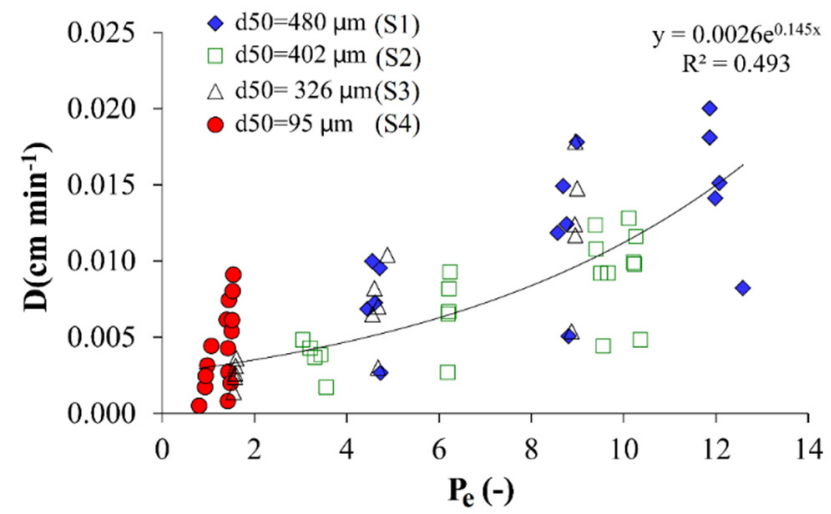

Fig. 10. Effect of the particle Peclet number $\left(P_{e}\right)$ on the dispersion coefficient $(D)$ for the different sand samples.

In contrast with $\mathrm{S} 1, \mathrm{~S} 2$ and $\mathrm{S} 3$, fine sand sample $\mathrm{S} 4$ showed relatively low Peclet numbers for all velocities $\left(P_{e}<3\right)$. This indicates that solute dispersion for that sample may have been affected also by molecular diffusion, in part because of the relatively low pore-water velocities for that sample. Results further showed that with an increase in the Peclet number and/or pore-water velocity, the dispersion coefficient increased nonlinearly, which is consistent with earlier studies (d'Angelo et al., 2007; Kantzas et al., 2012).

\section{Correlation matrix of parameters controlling the dispersion coefficient}

A correlation matrix of the various parameters controlling the dispersion coefficient is provided in Table 3 . The data indicate a significant correlation between $D$ and five parameters, namely the Peclet number $\left(P_{e}\right)$, the dispersivity $(\lambda)$, the pore- water velocity $(v)$, the travel distance $(L)$ and the average grain diameter $\left(d_{50}\right)$. Of these, three parameters $\left(v, \lambda\right.$, and $\left.P_{e}\right)$ are directly related to $D$ as expected (e.g., through Eq. 6), while the parameters of $L$ and Grain Size Variation (GSV) are independent variables. A strong-positive correlation was found between the dispersion coefficient and the Peclet number, the dispersivity, and the pore-water velocity as reflected by values of $0.709,0.674$, and 0.653 for the Pearson correlation coefficient, respectively (Evans, 1996). Pearson's correlation coefficient measures the statistical relationship, or association, between two continuous variables. We used the guidelines by Evans (1996) to evaluate the degree of positive and negative correlation, i.e., very weak $(0.00-0.019)$, weak $(0.20-0.39)$, moderate (0.40-0.59), strong $(0.60-0.79)$, or very strong $(0.80-1.0)$.

A moderate-positive correlation was found between $D$ and both the travel distance and the average grain diameter, having Pearson correlation coefficients of 0.546 and 0.454 , respectively. However, we found almost no correlation between $D$ and porosity $(\phi)$ and grain size variation $(G S V)$. As shown by Eq. (6), the Peclet number accounts for both the pore-water velocity (v) and the average grain diameter $\left(d_{50}\right)$. As such, it is not surprising that a strong-positive relationship was obtained between the Peclet number and $D$, leading to relatively high and low values of the Pearson correlation coefficient with $P_{e}$.

\section{CONCLUSIONS}

In this study we used two sand samples (S1 and S4 having $d_{50}$ values of about 480 and $95 \mu \mathrm{m}$, with narrow particle size distribution, respectively) and their two mixtures (S2 and S3 having $d_{50}$ values of 402 and $324 \mu \mathrm{m}$ with dual particle size distribution) to explore the effects of soil textural characteristics on longitudinal dispersion in $36-\mathrm{cm}$ long saturated sand columns. Breakthrough curves (BTCs) at 3 depths $(11,18,25 \mathrm{~cm})$ and of the effluent at $36 \mathrm{~cm}$ were obtained by measuring the electrical conductivity (EC) using 5TE sensors.

Analyses of the BTCs in terms of the macroscopic equilibrium advection-dispersion equation revealed that the dispersion coefficient $(D)$ of the sands increased with the pore-water velocity $(v)$. The $D$ value of sample S1 with its relatively large mean grain size was more affected by the pore-water velocity as indicated by much larger dispersivities $(\lambda)$, Furthermore, the $D$ values increased with travel distance to slowly approach asymptotic values at distances of 25 to $36 \mathrm{~cm}$. Our results revealed that for a constant velocity, the longitudinal dispersion coefficient of sample S1 (the coarser sand) was much higher than $D$ of sample $\mathrm{S} 4$ which had a small $d_{50}$. In addition to increasing with the pore-water velocity and travel distance, $D$ can be affected also by the grain size distribution. Our results showed that $D$ of sand sample S4 (the finer sand) was smaller than of the sample $\mathrm{S} 1$ at a same velocity.

Table 3. Correlation matrix of various transport and porous media parameters affecting the dispersion coefficient

\begin{tabular}{|c|c|c|c|c|c|c|c|}
\hline & ${ }^{1} \boldsymbol{D}$ & ${ }^{2} \boldsymbol{v}$ & ${ }^{3} \lambda$ & ${ }^{4} \boldsymbol{L}$ & ${ }^{5} d_{50}$ & ${ }^{6} \boldsymbol{G S V}$ & ${ }^{7} \phi$ \\
\hline $\boldsymbol{v}$ & $0.653^{* *}$ & & & & & & \\
\hline$\lambda$ & $0.674^{* *}$ & $-0.036^{\mathrm{ns}}$ & & & & & \\
\hline$L$ & $0.546^{* *}$ & $0.005^{\mathrm{ns}}$ & $0.72^{* *}$ & & & & \\
\hline$d_{50}$ & $0.454^{* *}$ & $0.24^{\mathrm{ns}}$ & $0.47^{* *}$ & $0^{\mathrm{ns}}$ & & & \\
\hline$G S V$ & $-0.007^{\mathrm{ns}}$ & $0.138^{\mathrm{ns}}$ & $-0.046^{\mathrm{ns}}$ & $0^{\mathrm{ns}}$ & $0.418^{*}$ & & \\
\hline$\phi$ & $-0.062^{\mathrm{ns}}$ & $-0.104^{\mathrm{ns}}$ & $-0.096^{\mathrm{ns}}$ & $0.026^{\mathrm{ns}}$ & $-0.506^{* *}$ & $-0.91^{* *}$ & \\
\hline${ }^{8} \boldsymbol{P}_{e}$ & $0.709^{* *}$ & $0.834^{* *}$ & $0.212^{\mathrm{ns}}$ & $-0.006^{\mathrm{ns}}$ & $0.716^{* *}$ & $0.296^{*}$ & $-0.314^{*}$ \\
\hline
\end{tabular}

${ }^{1}$ Dispersion coefficient; ${ }^{2}$ Pore-water velocity; ${ }^{3}$ Dispersivity; ${ }^{4}$ Travel distance; ${ }^{5}$ Average diameter of grains; ${ }^{6}$ Grain size variation; ${ }^{7}$ Porosity; ${ }^{8}$ Peclet Number; ${ }^{*} \mathrm{P} \leq 0.05$ (significant difference); ${ }^{* *} \mathrm{P} \leq 0.01$ (highly significant difference); ${ }^{\text {ns }} \mathrm{P}>0.05$ (no significant difference). 
We further found very large particle Peclet numbers for the coarser sand samples with their larger mean diameters (notably $\mathrm{S} 1$, but also S2, and S3). This confirms the validity of our assumption that the contribution of molecular diffusion was negligible. In contrast to S1, S2 and S3, the sample S4 (with its finer sand) showed a relatively low Peclet number $\left(P_{e}\right)$ for all velocities $\left(P_{e}<2\right)$. This indicates that dispersion in sample S4 was affected more by molecular diffusion. Our results also showed that with an increase in the Peclet number and/or porewater velocity, the dispersion coefficient increased nonlinearly, with an exponential shape.

A correlation matrix of the many parameters potentially affecting dispersion processes showed a strong-positive correlation between $D$ and three parameters: The Peclet number, the dispersivity, and the pore-water velocity, which is in line with Equations (5) and (6). A moderate-positive correlation between the dispersion coefficient and travel distance and the average diameter of the grains was found. However, almost no correlation existed between $D$ and the porosity as well as grain-size heterogeneity. Consequently, it seems that that the dispersion coefficient depends on particle size more than other soil texture or structure features (e.g., porosity, particle size distribution modality).

The results from this study suggest using scale-dependent relations for the solute dispersivity, particularly for applications where the scale- dependency of solute dispersion is important, such as at early times and/or short travel distances. We further note that our study was limited to saturated conditions. Several experimental (e.g., Bunsri et al., 2008b; Toride et al., 2003) and pore-scale modeling (e.g., Raoof and Hassanizadeh, 2013) studies suggest that the dispersivity may increase when soils become unsaturated up until some maximum at intermediate fluid saturation values, with lower values again in relatively dry conditions. Several studies also reported water fractionation into mobile and immobile water fractions (e.g., Gaudet et al., 1977). These and other effects such as heterogeneity and macropore flow processes also need further investigations at a range of scales.

\section{REFERENCES}

Bandai, T., Hamamoto, S., Rau, G.C., Komatsu, T., Nishimura, T., 2017. The effect of particle size on thermal and solute dispersion in saturated porous media. Int. J. Therm. Sci., $122,74-84$.

Bear, J., 1988. Dynamics of Fluids in Porous Media. Dover, New York.

Bolt, G.H., 1979. Movement of solutes in soil: Principles of adsorption/exchange chromatography. Chapter 9. In: Developments in Soil Science, Vol. 5, Part B, pp. 285-348.

Bromly, M., Hinz, C., Aylmore, L.A.G., 2007. Relation of dispersivity to properties of homogeneous saturated repacked soil columns. Eur. J. Soil Sci., 58, 293-301.

Bunsri, T., Sivakumar, M., Hagare, D., 2008a. Influence of dispersion on transport of tracer through unsaturated porous media. J. Appl. Fluid Mech., 1, 37-44.

Bunsri, T., Sivakumar, M., Hagare, D., 2008b. Numerical modelling of tracer transport in unsaturated porous media. J. Appl. Fluid Mech., 1, 1, 62-70.

Cui, X., Zhu, C., Hu, M., Wang, X., Liu, H., 2019. The hydrodynamic dispersion characteristics of coral sands. J. Mar. Sci. Eng., 7, 9, Article Number: 291.

d'Angelo, M.V., Auradou, H., Allain, C., Hulin, J.P., 2007. Pore scale mixing and macroscopic solute dispersion re- gimes in polymer flows inside two-dimensional model networks. Phys. Fluids, 19, 3, Article Number: 033103.

Delgado, J.M.P.Q., 2006. A critical review of dispersion in packed beds. Heat and Mass Transfer, 42, 4, 279-310.

Delgado, J.M.P.Q., 2007. Longitudinal and transverse dispersion in porous media. Chem. Eng. Res. Des., 85, 9, 12451252.

Dong, X., Liu, X., Woo, H., Park, J., 2014. A developed soil column test device for measuring the electrical conductivity breakthrough curves. Environ. Earth Sci., 72, 9, 3715-3722.

Evans, J.D., 1996. Straightforward Statistics for the Behavioral Sciences. Pacific Grove, CA: Brooks/Cole Pub. 1.

Fetter, C.W., 2008. Contaminant Hydrogeology. 2th Ed. Waveland Press, Inc.

Freeze, R.A., Cherry, J.A., 1979. Groundwater. Prentice-Hall, Englewood Cliffs.

Gaudet, J., Jegat, H., Vachaud, G., Wierenga, P., 1977. Solute transfer, with exchange between mobile and stagnant water, through unsaturated sand. Soil Sci. Soc. Am. J., 41, 4, 665671.

Godoy, V.A., Zuquette, L.V., Gómez-Hernández, J.J., 2019. Stochastic upscaling of hydrodynamic dispersion and retardation factor in a physically and chemically heterogeneous tropical soil. Stoch. Env. Res. Risk A, 33, 1, 201-216.

Goldobin, D.S., Maryshev, B.S., 2017. Hydrodynamic dispersion in porous media with macroscopic disorder of parameters. In: Journal of Physics Conference Series, 894, 1, Article Number: 012062.

Hilhorst, M.A., 2000. A pore water conductivity sensor. Soil Sci. Soc. Am. J., 64, 6, 1922-1925.

Hunt, A.G., Skinner, T.E., Ewing, R.P., Ghanbarian-Alavijeh, B., 2011. Dispersion of solutes in porous media. Eur. Phys. J. B, 80, 411-432

Inoue, M., Šimůnek, J., Shiozawa, S., Hopmans, J.W., 2000. Simultaneous estimation of soil hydraulic and solute transport parameters from transient infiltration experiments. Adv. Water Resour., 23, 7, 677-688.

Kantzas, A., Bryan, J., Taheri, S., 2012. Fundamentals of fluid flow in porous media. Pore size distribution. PERM Inc.

Kanzari, S., Hachicha, M., Bouhlila, R., 2015. Laboratory method for estimating solute transport parameters of unsaturated soils, American Journal of Geophysics, Geochemistry and Geosystems, 1, 4, 149-154.

Kutílek, M., Nielsen, D.R., 1994. Soil Hydrology. Catena Verlag, Cremlingen-Destedt, Germany.

Lee, M.J., Hwang, S.I., Ro, H.M., 2014. Interpreting the effect of soil texture on transport and removal of nitrate-N in saline coastal tidal flats under steady-state flow condition. Cont. Shelf Res., 84, 35-42.

Lewis, J., Sjostrom, J., 2010. Optimizing the experimental design of soil columns in saturated and unsaturated transport experiments. J. Contam. Hydrol., 115, 1-13.

Lindstrom, F.T., Haque, R., Freed, V.H., Boersma, L., 1967. The movement of some herbicides in soils: Linear diffusion and convection of chemicals in soils. Environmental Science and Technology, 1, 561-565.

Nimbi, I.M., Powers, S., 2003. Mass transfer correlations for nonaqueous phase liquid dissolution from region with high initial saturation. Water Resour. Res., 39, 2, Article Number: 1030. DOI: 10. 1029/2001WR000667

Nützmann, G., Maciejewski, S., Joswig, K., 2002. Estimation of water saturation dependence of dispersion in unsaturated porous media: experiments and modelling analysis. Advances in Water Resources, 25, 5, 565-576. 
Poulsen, T.G., Suwarnarat, W., Hostrup, M.K., Kalluri, P.N.V., 2008. Simple and rapid method for measuring gas dispersion in porous media: methodology and applications. Soil Sci., 173, 3, 169-174.

Pugliese, L., Poulsen, T.G., Straface, S., 2013. Gas-solute dispersivity ratio in granular porous media as related to particle size distribution and particle shape. Water Air Soil Pollut., 224, Article Number: 1691.

Raoof, A., Hassanizadeh, S.M., 2013. Saturation-dependent solute dispersivity in porous media: Pore-scale processes. Water Resour. Res., 49, 1943-1951.

Saffman, P.G., 1959. A theory of dispersion in a porous medium. J. Fluid Mech., 6, 3, 321-349.

Scheidegger, A.E., 1974. The Physics of Flow through Porous Media. University of Toronto Press, Toronto.

Scudiero, E., Berti, A., Teatini, P., Morari, F., 2012. Simultaneous monitoring of soil water content and salinity with a low-cost capacitance-resistance probe. Sensors, 12, 12, 17588-17607.

Sharma, P., Poulsen, T.G., 2010. Gas dispersion and immobile gas content in granular porous media: Effect of particle size nonuniformity. Soil Sci., 175, 9, 426-431.

Šimunek, J., van Genuchten, M.Th., Šejna, M., Toride, N., Leij, F.J., 2000. The STANMOD computer software for evaluating solute transport in porous media using analytical solutions of convection-dispersion equation. Version 2.0, IGWMC-TPS71. Int. Ground Water Modeling Center (IGWMC), Colorado School of Mines, Golden, Colorado, $32 \mathrm{p}$.

Toride, N., Leij, F.J., van Genuchten, M.T., 1999. The CXTFIT code for estimating transport parameters from laboratory or field tracer experiments. Version 2.1, Research Report 137. U.S. Salinity Laboratory, Riverside, CA.

Toride, N., Inoue, M., Leij, F.J., 2003. Hydrodynamic dispersion in unsaturated dune sand. Soil Sci. Soc. Am. J., 67, 3, 703-712.

van Genuchten, M.Th., Parker, J.C., 1984. Boundary conditions for displacement experiments through short laboratory soil columns. Soil Sci. Soc. Am. J., 48, 4, 703-708.

van Genuchten, M.Th., Wierenga, P.J., 1976. Mass transfer studies in sorbing porous media I. Analytical solutions. Soil Sci. Soc. Am. J., 40, 4, 473-480. DOI: 10.2136/sssaj1976.03615995004000040011x

van Genuchten, M.Th., Šimůnek, J., Leij, F.J., Toride, N., Šejna, M., 2012. STANMOD: Model use, calibration and validation. Transport, ASABE, 55, 4, 1353-1366.

Vanderborght, J., Vereecken, H., 2007. Review of dispersivities for transport modeling in soils. Vadose Zone J., 6, 1, 29-52.

Received 16 December 2019 Accepted 4 January 2021 